\title{
TITLE:
}

\section{Topological Objects in Holographic QCD}

$\operatorname{AUTHOR}(\mathrm{S})$ :

Suganuma, Hideo; Hori, Keiichiro

\section{CITATION:}

Suganuma, Hideo ...[et al]. Topological Objects in Holographic QCD. Physica Scripta 2020, 95(7): 074014.

\section{ISSUE DATE:}

2020-07

URL:

http://hdl.handle.net/2433/254354

\section{RIGHT:}

This is the Accepted Manuscript version of an article accepted for publication in Physica Scripta. IOP Publishing Ltd is not responsible for any errors or omissions in this version of the manuscript or any version derived from it. The Version of Record is available online at https://doi.org/10.1088/1402-4896/ab986c.; The full-text file will be made open to the public on 8 June 2021 in accordance with publisher's 'Terms and Conditions for Self-Archiving'.; この論文は出版社版て ありません。引用の際には出版社版をご確認ご利用ください。;This is not the published version. Please cite only the published version. 


\title{
Topological Objects in Holographic QCD
}

\author{
Hideo Suganuma \\ Department of Physics, Kyoto University, \\ Kitashirakawaoiwake, Sakyo, Kyoto 606-8502, Japan \\ E-mail: suganuma@scphys.kyoto-u.ac.jp

\section{Keiichiro Hori} \\ Department of Physics, Kyoto University, \\ Kitashirakawaoiwake, Sakyo, Kyoto 606-8502, Japan \\ E-mail: hori@ruby.scphys.kyoto-u.ac.jp
}

March 2020

\begin{abstract}
.
We study topological objects in holographic QCD based on the Sakai-Sugimoto model, which is constructed with $N_{c}$ D4 branes and $N_{f}$ D8/D8 branes in the superstring theory, and is infrared equivalent to $1+3$ dimensional massless QCD. Using the gauge/gravity duality, holographic QCD is described as $1+4$ dimensional $\mathrm{U}\left(N_{f}\right)$ gauge theory in flavor space with a background gravity, and its instanton solutions correspond to baryons. First, using the Witten Ansatz, we reduce holographic QCD into a $1+2$ dimensional Abelian Higgs theory in a curved space and consider its topological aspect. We numerically obtain the Abrikosov vortex solution and investigate single baryon properties. Second, we study a single meron and two merons in holographic QCD. The single meron carrying a half-integer baryon number is found to have an infinite energy also in holographic QCD. We propose a new-type baryon excitation of the two-merons oscillation in the extra-direction of holographic QCD.
\end{abstract}

\section{Introduction}

Quantum chromodynamics (QCD) has been established as the fundamental theory of the strong interaction. In spite of great success of perturbative QCD in high-energy regions, QCD exhibits strong-coupling nature beyond the perturbation in low-energy regions. Still now, to understand nonperturbative properties of QCD is one of the most important and difficult problems remaining in theoretical physics. As an interesting effective theory of nonperturbative QCD, holographic QCD was formulated using Dbranes [1] and the gauge/gravity duality [2] in the superstring theory, and has been developed for the Yang-Mills theory [3] and massless QCD [4, 5, 6, 7, 8, 9, 10, 11, 12, 13].

The superstring theory, which is well-defined in ten-dimensional space-time, has $\mathrm{D} p$-branes [1] as $(p+1)$-dimensional massive soliton-like objects of fundamental strings. 
On the surface of $N$ D-branes, there exists a $\mathrm{U}(N)$ gauge symmetry, and $\mathrm{U}(N)$ gauge fields appear from open strings. Around the massive $N$ D-branes, a supergravity field proportional to $N$ is created in ten-dimensional space-time. Since the gravity field depends on the distance from the D-brane, one more coordinate-dependence at least appears in the gravity side, which is called holography.

The D-brane generally leads to a SUSY system, reflecting superstring nature. In 1998, Witten [3] constructed non-SUSY gauge theories in this framework by imposing explicit SUSY breaking through $S^{1}$-compactification of D-branes with the periodic/antiperiodic boundary condition for bosons/fermions, in a similar manner to thermal SUSY breaking. The inverse radius of the $S^{1}$ is called as the Kaluza-Klein mass $M_{\mathrm{KK}}$, and the fermionic gaugino mass becomes $O\left(M_{\mathrm{KK}}\right)$. Then, non-SUSY gauge theories are formed on the compactified D-branes at larger scale than $1 / M_{\mathrm{KK}}$.

Actually, a four-dimensional non-SUSY U $\left(N_{c}\right)$ Yang-Mills theory is realized on $S^{1}$ compactified $N_{c}$ D4-branes, where only bosonic gauge fields $\mathcal{A}^{\mu}$ remain to be massless. On the other hand, the effect from $N_{c}$ D4-branes can be also described by the gravity field around it, under hypothesis of the gauge/gravity duality [2]. In fact, the Yang-Mills theory or the gauge sector of QCD can be constructed with the $S^{1}$-compactified $N_{c}$ D4branes, and it is transferred into a higher-dimensional gravity theory in the holographic framework. Owing to the strong-weak coupling duality, large- $N_{c}$ strong-coupling QCD is described with the weak-coupling gravity [2,3], and nonperturbative quantities of infrared QCD is calculable with the higher-dimensional classical gravitational theory.

\section{Construction of holographic QCD: the Sakai-Sugimoto model}

In 2005, Sakai and Sugimoto [4] constructed four-dimensional massless QCD using the D4/D8/ $\overline{\mathrm{D} 8}$ multi-D-brane system, which consists of spatially $S^{1}$-compactified $N_{c}$ D4branes attached with $N_{f}$ D8- $\overline{\mathrm{D} 8}$ pairs, as shown in Fig.1(a).

In holographic QCD, "color" and "flavor" are described as different physical objects, i.e., different D-branes: the D4 gives color and the D8 gives flavor. Here, gluons $\mathcal{A}^{\mu} \in \mathrm{su}\left(N_{c}\right)$ appear from 4-4 strings on the D4-brane, and (anti)quarks, which have both color and flavor, appear from 4-8 (4- $\overline{8})$ strings at the cross point between D4 and $\mathrm{D} 8(\overline{\mathrm{D} 8})$. This D-brane system has an $\mathrm{SU}\left(N_{c}\right)$ gauge symmetry and the exact chiral symmetry, and leads to four-dimensional QCD in the chiral limit [4] at the larger scale than $M_{\mathrm{KK}}^{-1}$, i.e., the radius of the $S^{1}$. In fact, at the infrared scale, this multi-D-brane system is described by $1+3$ dimensional massless QCD:

$$
\mathcal{L}=-\frac{1}{2} \operatorname{tr}\left(\mathcal{F}_{\mu \nu} \mathcal{F}^{\mu \nu}\right)+\bar{q} i \gamma_{\mu} \mathcal{D}^{\mu} q
$$

Here, $\mathcal{F}^{\mu \nu}$ and $\mathcal{D}^{\mu}$ are the field strength and the covariant derivative operating in the color space,

$$
\mathcal{F}^{\mu \nu} \equiv \frac{1}{i g}\left[\mathcal{D}^{\mu}, \mathcal{D}^{\nu}\right], \quad \mathcal{D}^{\mu} \equiv \partial^{\mu}+i g \mathcal{A}^{\mu}
$$

with the QCD gauge coupling $g$. 
In large- $N_{c}$ argument, $N_{c}$ D4-branes have a large mass proportional to $N_{c}$, which gives a large gravitational source. Using the gauge/gravity duality, $N_{c}$ D4-branes can be replaced by a bulk gravity field, and the system becomes $N_{f}$ D8-branes in the presence of the background gravity of the $N_{c}$ D4-branes, as shown in Fig.1(b). In large $N_{c}\left(\gg N_{f}\right)$, the gravitational contribution from $\mathrm{D} 8 / \overline{\mathrm{D} 8}$ can be neglected, which corresponds to the quenched approximation [5].

In this framework, large 't Hooft's coupling $\lambda \equiv g^{2} N_{c}$ is taken, which means strongcoupling QCD and a weak-coupling gravity, due to the strong-weak duality. In large $N_{c}$ and large 't Hooft's coupling $\lambda$, the $N_{f}$ D8-brane system in the D4 background gravity can be expressed with the Dirac-Born-Infeld (DBI) action in nine-dimensional space-time $[1,4]$,

$$
S_{\mathrm{D} 8}^{\mathrm{DBI}}=T_{8} \int d^{9} x e^{-\phi} \operatorname{tr}\left\{\left[-\operatorname{det}\left(g_{M N}+2 \pi \alpha^{\prime} F_{M N}\right)\right]^{1 / 2}\right\},
$$

where $F_{M N}$ is the field strength in the $\mathrm{U}\left(N_{f}\right)$ flavor space on the D8 brane, and the $\mathrm{U}\left(N_{f}\right)$ gauge field originates from 8-8 strings. $T_{8}, \phi$ and $\alpha^{\prime}$ are quantities defined in the superstring theory [4]. Here, flavor degrees of freedom only remains, since the D4-brane with color is already replaced by the background gravity of $g_{M N}$.

This system possesses an $\mathrm{SO}(5)$ rotational symmetry in nine-dimensional spacetime, and hence the DBI action is reduced to be five-dimensional. In the leading of large $N_{c}$ and $\lambda$, the DBI action eventually becomes a five-dimensional $\mathrm{U}\left(N_{f}\right)$ flavored YangMills theory [4] with a curved metric on an extra fifth-coordinate $w$, which corresponds to the distance from the D4-brane.
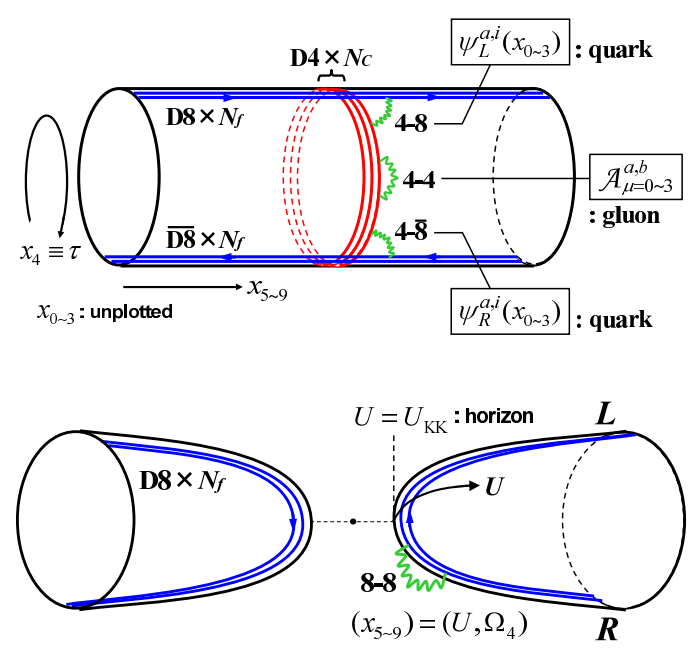

Figure 1. The Sakai-Sugimoto model. (a) The upper figure shows the multi-D-brane configuration corresponding to massless QCD: spatially $S^{1}$-compactified $N_{c}$ D4-branes attached with $N_{f}$ D8-D8 pairs. Gluons appear from 4-4 strings, and quarks appear from 4-8 and 4- $\overline{8}$ strings. (b) The lower figure shows the D8-brane with D4 background gravity, which is generated from $N_{c}$ D4-branes as a result of the gauge/gravity duality. Mesons appear from 8-8 strings. 
In this way, from the multi-D-brane system which is infrared equivalent to massless QCD, one derives $1+4$ dimensional Yang-Mills theory on $\mathrm{U}\left(N_{f}\right) \simeq \mathrm{SU}\left(N_{f}\right) \times \mathrm{U}(1)$ in the leading order of $1 / N_{c}$ and $1 / \lambda$ expansions:

$$
\begin{aligned}
S_{5 \mathrm{YM}}= & S_{5 \mathrm{YM}}^{\mathrm{SU}\left(N_{f}\right)}+S_{5 \mathrm{YM}}^{\mathrm{U}(1)} \\
= & -\kappa \int d^{4} x d w \operatorname{tr}\left[\frac{1}{2} h(w) F_{\mu \nu} F^{\mu \nu}+k(w) F_{\mu w} F^{\mu w}\right] \\
& -\frac{\kappa}{2} \int d^{4} x d w \operatorname{tr}\left[\frac{1}{2} h(w) \hat{F}_{\mu \nu} \hat{F}^{\mu \nu}+k(w) \hat{F}_{\mu w} \hat{F}^{\mu w}\right] .
\end{aligned}
$$

For $M, N=t, x, y, z, w$, the field strength is given by

$$
\begin{aligned}
& F_{M N} \equiv \partial_{M} A_{N}-\partial_{N} A_{M}+i\left[A_{M}, A_{N}\right], \\
& \hat{F}_{M N} \equiv \partial_{M} \hat{A}_{N}-\partial_{N} \hat{A}_{M}
\end{aligned}
$$

with the five-dimensional SU $\left(N_{f}\right)$ gauge field $A^{M}\left(x^{\mu}, w\right)$ and $\mathrm{U}(1)$ gauge field $\hat{A}^{M}\left(x^{\mu}, w\right)$. Here, we take the $M_{\mathrm{KK}}=1$ unit and $\kappa=\frac{\lambda N_{c}}{216 \pi^{3}}$. In Eq.(4), $\mathrm{SU}\left(N_{f}\right)$ variables $A$ and $\mathrm{U}(1)$ variables $\hat{A}$ are completely separated, and hence $S_{5 \mathrm{YM}}$ is divided into the $\mathrm{SU}\left(N_{f}\right)$ sector $S_{5 \mathrm{YM}}^{\mathrm{SU}\left(N_{f}\right)}$ and the U(1) sector $S_{5 \mathrm{YM}}^{\mathrm{U}(1)}$. Note that there appears background gravities $k(w)$ and $h(w)$ depending on the extra fifth-coordinate $w$,

$$
k(w)=1+w^{2}, \quad h(w)=k(w)^{-1 / 3},
$$

as a relic of $N_{c}$ D4-branes.

The $1 / N_{c}$-leading holographic QCD has also the Chern-Simons (CS) term $[4,6]$ as the next leading order of $1 / \lambda$. The CS term is a topological term responsible to anomalies in QCD, and its explicit form is

$$
\begin{aligned}
S_{\mathrm{CS}}= & \frac{N_{c}}{24 \pi^{2}} \int\left[\frac{3}{2} \hat{A} \operatorname{tr} F^{2}+\frac{1}{4} \hat{A} \hat{F}^{2}+(\text { total derivatives })\right] \\
= & \frac{N_{C}}{24 \pi^{2}} \epsilon_{M N P Q} \int d^{4} x d w\left[\frac{3}{8} \hat{A}_{0} \operatorname{tr} F_{M N} F_{P Q}-\frac{3}{2} \hat{A}_{M} \operatorname{tr}\left(\partial_{0} A_{N} F_{P Q}\right)\right. \\
& \left.+\frac{3}{4} \hat{F}_{M N} \operatorname{tr}\left(A_{0} F_{P Q}\right)+\frac{1}{16} \hat{A}_{0} \hat{F}_{M N} \hat{F}_{P Q}-\frac{1}{4} \hat{A}_{M} \hat{F}_{0 N} \hat{F}_{P Q}\right],
\end{aligned}
$$

where $A$ is the $\mathrm{SU}\left(N_{f}\right)$ gauge field and $\hat{A}$ the $\mathrm{U}(1)$ gauge field. Here and hereafter, the capital-letter index denotes the spatial index as $M=x, y, z, w$. In Eq.(7), $\mathrm{SU}\left(N_{f}\right)$ variables $A$ and $\mathrm{U}(1)$ variables $\hat{A}$ are dynamically mixed in $S_{\mathrm{CS}}$.

To summarize, in the Sakai-Sugimoto model, holographic QCD is expressed by $S_{\mathrm{HQCD}}=S_{5 \mathrm{YM}}+S_{\mathrm{CS}}$, up to the $1 / N_{c}$ leading and the $1 / \lambda$ next leading. Note that holographic QCD has a mathematical connection to infrared QCD through D-branes, and is successful to explain many phenomenological laws in hadron physics [4]: the vector-meson dominance (VMD), the Kawarabayashi-Suzuki-Riazuddin-Fayyazuddin (KSRF) relation, the Gell-Mann-Sharp-Wagner (GSW) relation, the hidden localsymmetry (HLS) picture, the Skyrme chiral-soliton picture [14, 15, 16] and so on. 


\section{Baryons from instantons in holographic QCD}

In holographic QCD, baryons appear from instantons in the flavor space [4, 6], and eventually they are described as chiral solitons [5]. Note here that holographic QCD already includes four-dimensional spatial coordinates $(x, y, z, w)$ including the extra fifth-coordinate $w$, and instantons can be naturally introduced in holographic QCD without necessity of the Euclidean process or the Wick rotation.

Actually, holographic QCD has instantons as topological solitons corresponding to the nontrivial homotopy group $\Pi_{3}\left(\mathrm{SU}\left(N_{f}\right)\right)=\mathbf{Z}$ in spatial coordinates $(x, y, z, w)[6,7]$, and the baryon number $B$ is expressed by the Pontryagin index [17],

$$
B=\frac{1}{16 \pi^{2}} \int d^{3} x d w \operatorname{tr}\left(F_{M N} \tilde{F}_{M N}\right) .
$$

After the mode expansion along the extra $w$-direction, holographic QCD becomes the 1+3 dimensional Skyrme model including vector and axial-vector mesons [4], and baryons eventually become topological hedgehog solitons corresponding to the nontrivial homotopy group $\Pi_{3}\left(\mathrm{SU}\left(N_{f}\right)_{L} \times \mathrm{SU}\left(N_{f}\right)_{R} / \mathrm{SU}\left(N_{f}\right)_{V}\right)=\mathbf{Z}$.

In 2006, we studied baryons as brane-induced Skyrmions in holographic QCD for $N_{f}=2$ in the leading of $1 / N_{c}$ and $1 / \lambda[5]$, where we have included the background gravity precisely, but considered only light-mesons (pions and $\rho$-mesons) contributions. In 2007, Hata et al. described baryons from instantons in holographic QCD for $N_{f}=2$ [6], but they neglected the background gravity $h(w)$ and $k(w)$, because of the difficulty to deal with instantons in the curved space. In 2014, Bolognesi et al. numerically investigated the holographic baryon, precisely keeping the background gravity, using the $\mathrm{SO}(3)$ rotational symmetry $[11,12]$.

\subsection{Witten Ansatz for holographic $Q C D$}

To begin with, in a similar manner in Refs.[11, 12], we investigate baryons from instantons in the curved space with the background gravity $h(w)$ and $k(w)$ in the framework of $1+4$ dimensional holographic QCD for $N_{f}=2$. The strategy is to use the Witten Ansatz [18], which keeps the spatially rotational $\mathrm{SO}(3)$ symmetry on $\mathbf{x} \equiv(x, y, z)$ and $\mathbf{A} \equiv\left(A_{x}, A_{y}, A_{z}\right)$.

For the $\mathrm{SU}\left(N_{f}=2\right)$ gauge field $A=A^{a} \frac{\tau^{a}}{2} \in \mathrm{su}(2)_{f}$ in holographic QCD, we take the Witten Ansatz [18]:

$$
\begin{aligned}
& A_{0}^{a}(t, x, y, z, w)=a_{0}(t, r, w) \hat{x}^{a}, \\
& A_{i}^{a}(t, x, y, z, w)=\frac{\phi_{2}(t, r, w)+1}{r} \epsilon_{i a k} \hat{x}_{k}+\frac{\phi_{1}(t, r, w)}{r} \hat{\delta}_{i a}+a_{r}(t, r, w) \hat{x}_{i} \hat{x}_{a}, \\
& A_{w}^{a}(t, x, y, z, w)=a_{w}(t, r, w) \hat{x}^{a},
\end{aligned}
$$

with $r \equiv\left(x_{i} x_{i}\right)^{1 / 2}, \hat{x}_{i} \equiv x_{i} / r$ and $\hat{\delta}_{i j} \equiv \delta_{i j}-\hat{x}_{i} \hat{x}_{j}$. Note that this form has the $\operatorname{SO}(3)$ symmetry on the spatial rotation of $\mathbf{x} \rightarrow R \mathbf{x}$ and $\mathbf{A} \rightarrow R \mathbf{A}$, together with the isospin rotation $\vec{\tau} \rightarrow R \vec{\tau}$, for arbitrary $\mathrm{SO}(3)$ matrix $R$. This symmetry keeps the hedgehog structure $\tau^{a} \hat{x}^{a}$ invariant, and is useful in describing topological solitons. 
By way of the Witten Ansatz, $1+4$ dimensional $\mathrm{SU}(2)_{f}$ holographic QCD is reduced into a $1+2$ dimensional Abelian Higgs theory on a curved space. In fact, the leading term $S_{5 \mathrm{YM}}^{\mathrm{SU}(2)_{f}}$ is rewritten as

$$
\begin{aligned}
S_{5 \mathrm{YM}}^{\mathrm{SU}(2)_{f}}= & -\kappa \int d^{4} x d w \operatorname{tr}\left[\frac{1}{2} h(w) F_{\mu \nu} F^{\mu \nu}+k(w) F_{\mu w} F^{\mu w}\right] \\
= & 4 \pi \kappa \int_{-\infty}^{\infty} d t \int_{0}^{\infty} d r \int_{-\infty}^{\infty} d w\left[h(w)\left(\left|D_{0} \phi\right|^{2}-\left|D_{1} \phi\right|^{2}\right)-k(w)\left|D_{2} \phi\right|^{2}\right. \\
& \left.-\frac{h(w)}{2 r^{2}}\left(1-|\phi|^{2}\right)^{2}+\frac{r^{2}}{2}\left\{h(w) f_{01}^{2}+k(w) f_{02}^{2}-k(w) f_{12}^{2}\right\}\right]
\end{aligned}
$$

where the Abelian Higgs field $\phi(t, r, w) \in \mathbf{C}$, the Abelian gauge field $a_{\mu}(t, r, w)$, the covariant derivative $D_{\mu}$ and the field strength $f_{\mu \nu}$ in the Abelian Higgs theory are

$$
\phi \equiv \phi_{1}+i \phi_{2}, a_{\mu} \equiv\left(a_{0}, a_{r}, a_{w}\right), D_{\mu} \equiv \partial_{\mu}-i a_{\mu}, f_{\mu \nu} \equiv \partial_{\mu} a_{\nu}-\partial_{\nu} a_{\mu} .
$$

Here, we have used $(0,1,2)=(t, r, w)$ for the index of $1+2$ dimensional coordinates.

\subsection{Vortex description of baryon}

Here, we investigate the topological correspondence between the above two theories, by considering the topological charge corresponding to the baryon number $B$. In holographic QCD, instantons are responsible to the baryon number [4, 6]. Remarkably, the instanton in holographic QCD is described as the Abrikosov vortex in the $1+2$ dimensional Abelian Higgs theory [18], as is explicitly shown below.

For the $\mathrm{SU}(2)_{f}$ configuration in the Witten Ansatz, the topological density $\rho_{B}$ in $(x, y, z, w)$-space is given by

$$
\begin{aligned}
\rho_{B} & \equiv \frac{1}{16 \pi^{2}} \operatorname{tr}\left(F_{M N} \tilde{F}_{M N}\right)=\frac{1}{32 \pi^{2}} \epsilon_{M N P Q} \operatorname{tr}\left(F_{M N} F_{P Q}\right) \\
& =\frac{1}{8 \pi^{2} r^{2}} \epsilon_{i j} \partial_{i}\left\{a_{j}\left(1-|\phi|^{2}\right)+\partial_{j} \theta \cdot|\phi|^{2}\right\},
\end{aligned}
$$

where $\theta \equiv \arg \phi$ and the Roman indices $i, j$ take $(1,2)=(r, w)$. The topological density $\rho_{B}$ is expressed as a total derivative. Note that $\rho_{B}$ is independent from the spatial direction $(\hat{x}, \hat{y}, \hat{z})$, and thus takes an $\mathrm{SO}(3)$ rotationally symmetric form of $\rho_{B}(r, w)$.

Then, the baryon number $B$ or the Pontryagin index is written by a contour integral in $(r, w)$-plane,

$$
\begin{aligned}
B & =\int d^{3} x d w \rho_{B}=\frac{1}{2 \pi} \int_{0}^{\infty} d r \int_{-\infty}^{\infty} d w \epsilon_{i j} \partial_{i}\left\{a_{j}\left(1-|\phi|^{2}\right)+\partial_{j} \theta \cdot|\phi|^{2}\right\} \\
& =\oint_{r \geq 0} d \mathbf{s} \cdot\left\{\mathbf{a}\left(1-|\phi|^{2}\right)+\nabla \theta \cdot|\phi|^{2}\right\}=\oint_{r \geq 0} d \mathbf{s} \cdot \nabla \theta,
\end{aligned}
$$

where $\oint_{r \geq 0}$ denotes the contour integral around the whole half-plane of $(r, w)$ with $r \geq 0$. Here, we have used $\left|\phi\left(r=0,{ }^{\forall} w\right)\right|=1$ and $|\phi(\infty)|=1$ in $(r, w)$-plane, as the boundary condition for the finite-energy solution. (See Eqs.(10) and (14).) Thus, the baryon number $B$ is converted into the vortex number in this formalism. 


\subsection{Baryons in leading order of $1 / N_{c}$ and $1 / \lambda$}

In the reduced low-dimensional Abelian Higgs theory, the energy of the static configuration is simply expressed in the temporal gauge $a_{0}=0$ by

$$
\begin{aligned}
E_{5 \mathrm{YM}}^{\mathrm{SU}(2)_{f}}= & 4 \pi \kappa \int_{0}^{\infty} d r \int_{-\infty}^{\infty} d w\left[h(w)\left|D_{1} \phi\right|^{2}+k(w)\left|D_{2} \phi\right|^{2}\right. \\
& \left.+\frac{h(w)}{2 r^{2}}\left(1-|\phi|^{2}\right)^{2}+\frac{r^{2}}{2} k(w) f_{12}^{2}\right] .
\end{aligned}
$$

In the leading order of $1 / N_{c}$ and $1 / \lambda$, one only has to consider the main $\mathrm{SU}\left(N_{f}\right)$ part, $S_{5 \mathrm{YM}}^{\mathrm{SU}(2)_{f}}$ and $E_{5 \mathrm{YM}}^{\mathrm{SU}(2)_{f}}$, in holographic QCD, since the U(1) part is completely separated in $S_{5 \mathrm{YM}}$ in Eq.(4). Furthermore, the classical-level analysis is enough meaningful, because this theory is weak-coupling corresponding to strong-coupling QCD via the strongweak duality. Nawa et al. [5] investigated baryons as the brane-induced Skyrmions by truncating the higher mesonic modes than $M_{\mathrm{KK}}$, and obtained finite-size baryons from the DBI action $S_{5 \mathrm{YM}}$. However, if no truncation is done for the mesonic modes in $S_{5 \mathrm{YM}}$, the instanton solution or the ground-state baryon shrinks to be point-like [6], which means that the derivative becomes quite large and the low-energy treatment is to be corrected. As a remedy, Hata et al. [6, 7] included the Chern-Simons (CS) term, which is the next leading order of the $1 / \lambda$ expansion, and obtained a finite-size instanton for the baryon.

Here, in the leading order of $1 / N_{c}$ and $1 / \lambda$, we numerically calculate the groundstate baryon in Eq.(14), and also find the shrinking of the Abrikosov vortex solution into the point $(r, w)=(0,0)$, which corresponds to a point-like instanton at $w=0[18]$.

\section{4. $U(1)$ sector in holographic $Q C D$}

In the leading order of $1 / N_{c}$ and $1 / \lambda$, the $\mathrm{U}(1)$ sector is decoupled with the $\mathrm{SU}\left(N_{f}\right)$ sector, and has no physical importance. At the next leading order of $1 / \lambda$, however, the $\mathrm{U}(1)$ sector is coupled with the $\mathrm{SU}\left(N_{f}\right)$ sector through the CS term, and has physical importance [6].

Also for the $\mathrm{U}(1)$ gauge field $\hat{A}$, we respect the spatial $\mathrm{SO}(3)$ rotational symmetry $[11,12]$ as in the Witten Ansatz, and impose

$$
\hat{A}_{i}(t, x, y, z, w)=\hat{a}_{r}(t, r, w) \hat{x}_{i},
$$

while $\hat{A}_{0}$ and $\hat{A}_{w}$ are treated to be arbitrary. In this case, one finds $\hat{F}_{i j}=0$ and can take the $\hat{a}_{r}=0$ gauge. Then, the $1 / \lambda$-leading term $S_{5 \mathrm{YM}}^{\mathrm{U}(1)}$ is written as

$$
\begin{aligned}
S_{5 \mathrm{YM}}^{\mathrm{U}(1)} & =\frac{\kappa}{2} \int d^{4} x d w\left\{h(w) \hat{F}_{0 i}^{2}+k(w) \hat{F}_{0 w}^{2}-k(w) \hat{F}_{i w}^{2}\right\} \\
& =\int d^{4} x d w\left[\frac{1}{2} \hat{A}_{0} K \hat{A}_{0}-\frac{\kappa}{2} k(w)\left(\partial_{i} \hat{A}_{w}\right)^{2}\right],
\end{aligned}
$$

using the $\mathrm{SO}(3)$-symmetric non-negative hermite kernel

$$
K \equiv-\kappa\left\{h(w) \partial_{i}^{2}+\partial_{w} k(w) \partial_{w}\right\}=-\kappa\left\{h(w) \frac{1}{r^{2}} \partial_{r} r^{2} \partial_{r}+\partial_{w} k(w) \partial_{w}\right\} .
$$




\subsection{Baryons up to next leading order of $1 / \lambda$}

Next, let us consider the CS term $S_{\mathrm{CS}}$ as the next leading order of the $1 / \lambda$ expansion. For the static $\mathrm{SO}(3)$-rotationally symmetric configuration in the $A_{0}=0$ gauge, the CS term $S_{\mathrm{CS}}$ in Eq. $(7)$ is simply expressed as $[6,11,12]$

$$
S_{\mathrm{CS}}=\frac{N_{c}}{2} \int d^{4} x d w \rho_{B} \hat{A}_{0}
$$

which is the Coulomb-type interaction between the $\mathrm{U}(1)$ gauge potential $\hat{A}_{0}$ and the topological density $\rho_{B} \equiv \frac{1}{16 \pi^{2}} \operatorname{tr}\left(F_{M N} \tilde{F}_{M N}\right)$.

Then, the total $\mathrm{U}(1)$ action depending on the $\mathrm{U}(1)$ gauge field $\hat{A}$ is written as

$$
\begin{aligned}
S^{\mathrm{U}(1)} & \equiv S_{5 \mathrm{YM}}^{\mathrm{U}(1)}+S_{\mathrm{CS}} \\
& =\int d^{4} x d w\left[\frac{1}{2} \hat{A}_{0} K \hat{A}_{0}+\frac{N_{c}}{2} \rho_{B} \hat{A}_{0}-\frac{\kappa}{2} k(w)\left(\partial_{i} \hat{A}_{w}\right)^{2}\right],
\end{aligned}
$$

which leads to the field equations,

$$
K \hat{A}_{0}+\frac{N_{c}}{2} \rho_{B}=0, \quad \partial_{i}^{2} \hat{A}_{w}=0 .
$$

Note again that the classical-level analysis is enough meaningful, because of the weak coupling corresponding to strong-coupling QCD.

Thus, for the static solution, we obtain from $S^{\mathrm{U}(1)}$ the additional energy term

$$
\begin{aligned}
E^{\mathrm{U}(1)} & =\frac{N_{c}^{2}}{8} \int d^{3} x d w \rho_{B} K^{-1} \rho_{B} \\
& =\frac{N_{c}^{2}}{8} \int d^{3} x d w \int d^{3} x^{\prime} d w^{\prime} \rho_{B}(\vec{x}, w) K^{-1}\left(\vec{x}, w ; \vec{x}^{\prime}, w^{\prime}\right) \rho_{B}\left(\vec{x}^{\prime}, w^{\prime}\right),
\end{aligned}
$$

apart from the leading energy term $E_{5 \mathrm{YM}}^{\mathrm{SU}(2)_{f}}$. This additional term $E^{\mathrm{U}(1)}$ is the repulsive Coulomb-type energy among the topological density $\rho_{B}$, and this repulsive force prevents the spatial gathering of the topological-charge distribution [6].

Since the kernel $K$ and the topological density $\rho_{B}$ are $\mathrm{SO}(3)$-rotationally symmetric, the additional energy is expressed only with the $(r, w)$-coordinates:

$$
\begin{aligned}
& E^{\mathrm{U}(1)}=2 \pi^{2} N_{c}^{2} \int_{0}^{\infty} d r \int_{-\infty}^{\infty} d w \int_{0}^{\infty} d r^{\prime} \int_{-\infty}^{\infty} d w^{\prime} \\
& \tilde{\rho}(r, w) \tilde{K}^{-1}\left(r, w ; r^{\prime}, w^{\prime}\right) \tilde{\rho}\left(r^{\prime}, w^{\prime}\right),
\end{aligned}
$$

using $\tilde{\rho}(r, w) \equiv r^{2} \rho_{B}(r, w)$ and the hermite kernel $\tilde{K}$ in $(r, w)$-space,

$$
\tilde{K} \equiv 4 \pi r^{2} K=-4 \pi \kappa\left\{h(w) \partial_{r} r^{2} \partial_{r}+r^{2} \partial_{w} k(w) \partial_{w}\right\} .
$$

For the numerical calculation, we consider the total energy $E \equiv E_{5 \mathrm{YM}}^{\mathrm{SU}(2)_{f}}+E^{\mathrm{U}(1)}$ corresponding to $S_{\mathrm{HQCD}}=S_{5 \mathrm{YM}}+S_{\mathrm{CS}}$, and obtain the $B=1$ vortex solution on latticediscretized $(r, w)$-plane. Owing to the $\mathrm{SO}(3)$ rotational symmetry, the numerical cost is significantly reduced from $(4 \mathrm{D} \text { volume })^{2}$ to $(2 \mathrm{D} \text { volume })^{2}$. 


\subsection{Abrikosov vortex solution for baryon in holographic $Q C D$}

Now, we show the numerical result for the vortex solution for the baryon with $B=1$. As for the two parameters $M_{\mathrm{KK}}$ and $\kappa$ in holographic QCD, we take $M_{\mathrm{KK}} \simeq 948 \mathrm{MeV}$ and $\kappa=7.46 \times 10^{-3}$, to reproduce $f_{\pi} \simeq 92.4 \mathrm{MeV}$ and $m_{\rho} \simeq 776 \mathrm{MeV}[4,5]$.

Figure 2 shows the Higgs-field configuration $\vec{\phi}=\left(\phi_{1}, \phi_{2}\right)$ and the Abelian gauge configuration $\vec{a}=\left(a_{r}, a_{w}\right)$ in $(r, w)$-plane for the static Abrikosov vortex solution in the $1+2$ dimensional Abelian Higgs theory in the Landau gauge, $\partial_{r} a_{r}+\partial_{w} a_{w}=0$.
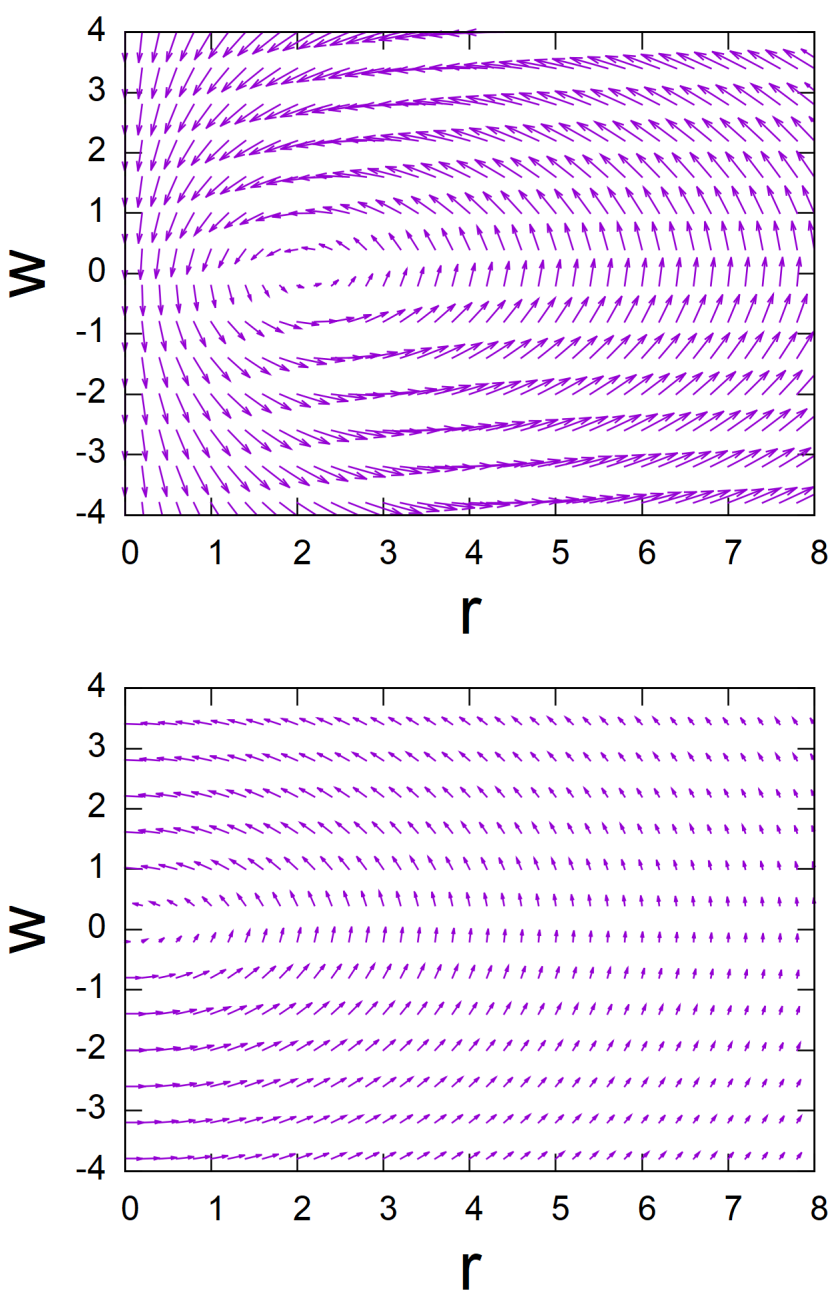

Figure 2. The static Abrikosov vortex solution for the baryon with $B=1$ in the $1+2$ Abelian Higgs theory reduced from $1+4$ holographic QCD using the Witten Ansatz. The upper figure shows the Higgs-field configuration $\vec{\phi}=\left(\phi_{1}, \phi_{2}\right)$, and the lower figure the Abelian gauge configuration $\vec{a}=\left(a_{r}, a_{w}\right)$ in $(r, w)$-plane in the Landau gauge. As the topological structure, the single winding number around $\left(r_{0}, w_{0}\right) \simeq(2,0)$, the zero point of the Higgs field $\vec{\phi}$, corresponds to the single baryon number $B=1$. The axis unit is $M_{\mathrm{KK}}^{-1} \simeq 0.2 \mathrm{fm}$.

In Fig.2, the Higgs field $\vec{\phi}(r, w)$ has the zero point at $(r, w)=\left(r_{0}, w_{0}\right) \simeq(2,0)$, and forms the topological structure of the Abrikosov vortex around it. In fact, we find the 
single winding number around the vortex center $\left(r_{0}, w_{0}\right)$, and this corresponds to the single baryon number $B=1$, as shown in Eq.(13).

Since the $r$-coordinate of the vortex center corresponds to the instanton size in the Witten Ansatz [18], from the vortex center $\left(r_{0}, w_{0}\right) \simeq(2,0)$, the instanton size $a$ is estimated as $a=r_{0}$, i.e., $a=r_{0} M_{\mathrm{KK}}^{-1} \simeq 0.4 \mathrm{fm}$ in the physical unit.

Figure 3 shows the topological density $\rho_{B}(r, w)$ and the energy density $\mathcal{E}(r, w)$ in $(r, w)$-plane for the Abrikosov vortex solution. For both topological and energy densities, we find an extended lump around the vortex center. Here, the integrated topological density is the baryon number $B$, and the baryon mass $M_{B}$ is given by the integrated energy density as the static energy of the ground-state baryon:

$$
\begin{aligned}
B & =\int d^{3} x d w \rho_{B}=4 \pi \int_{0}^{\infty} d r r^{2} \int_{-\infty}^{\infty} d w \rho_{B}(r, w) \\
M_{B} & =\int d^{3} x d w \mathcal{E}(r, w)=4 \pi \int_{0}^{\infty} d r r^{2} \int_{-\infty}^{\infty} d w \mathcal{E}(r, w) .
\end{aligned}
$$
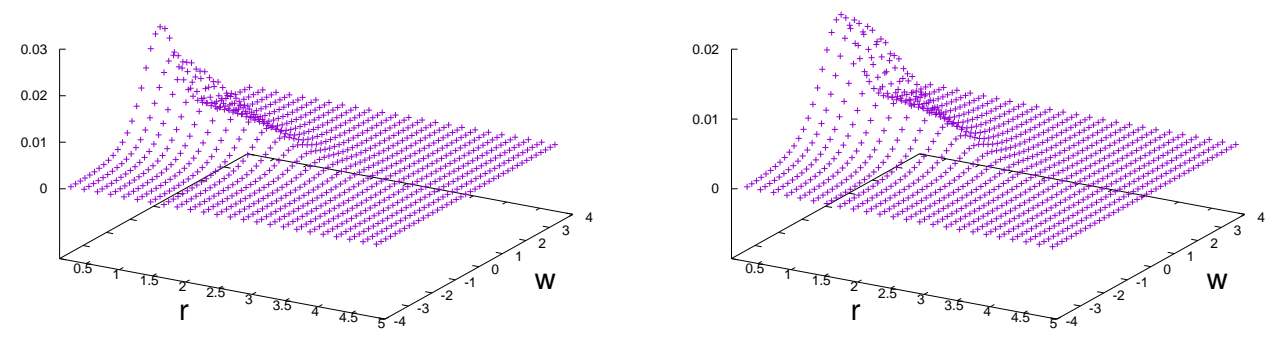

Figure 3. The left and right figures show the topological density $\rho_{B}(r, w)$ and the energy density $\mathcal{E}(r, w)$, respectively, in $(r, w)$-space for the Abrikosov vortex solution in the $1+2$ Abelian Higgs theory, reduced from $1+4$ holographic QCD. The scale unit is $M_{\mathrm{KK}}=1$. The integrated topological density is the baryon number $B=1$.

The ordinary energy and baryon-number densities in the three-dimensional space are obtained by the integration over the extra coordinate $w$ as

$$
\mathcal{E}(r) \equiv \int_{-\infty}^{\infty} d w \mathcal{E}(r, w), \quad \rho_{B}(r) \equiv \int_{-\infty}^{\infty} d w \rho_{B}(r, w)
$$

We show in Fig. 4 the energy density $\mathcal{E}(r)$ and the baryon-number density $\rho_{B}(r)$ inside the baryon as the function of $r$ in holographic QCD.

Thus, the baryon mass $M_{B}$ and the baryon size are estimated as

$$
M_{B}=\int d^{3} x \mathcal{E}(r) \simeq 998 \mathrm{MeV}, \sqrt{\left\langle r^{2}\right\rangle} \equiv \sqrt{\frac{\int d^{3} x \mathcal{E}(r) r^{2}}{\int d^{3} x \mathcal{E}(r)}} \simeq 0.48 \mathrm{fm} .
$$

In a similar manner in the Skyrme model [16], to perform semi-classical quantization of the baryonic soliton might be interesting and important $[6,8]$, but such a mass correction from the rotational energy of the soliton is only $O\left(1 / N_{c}\right)$ and is much higher order in the $1 / N_{c}$ expansion, in comparison with the baryon mass of $O\left(N_{c}\right)$. 


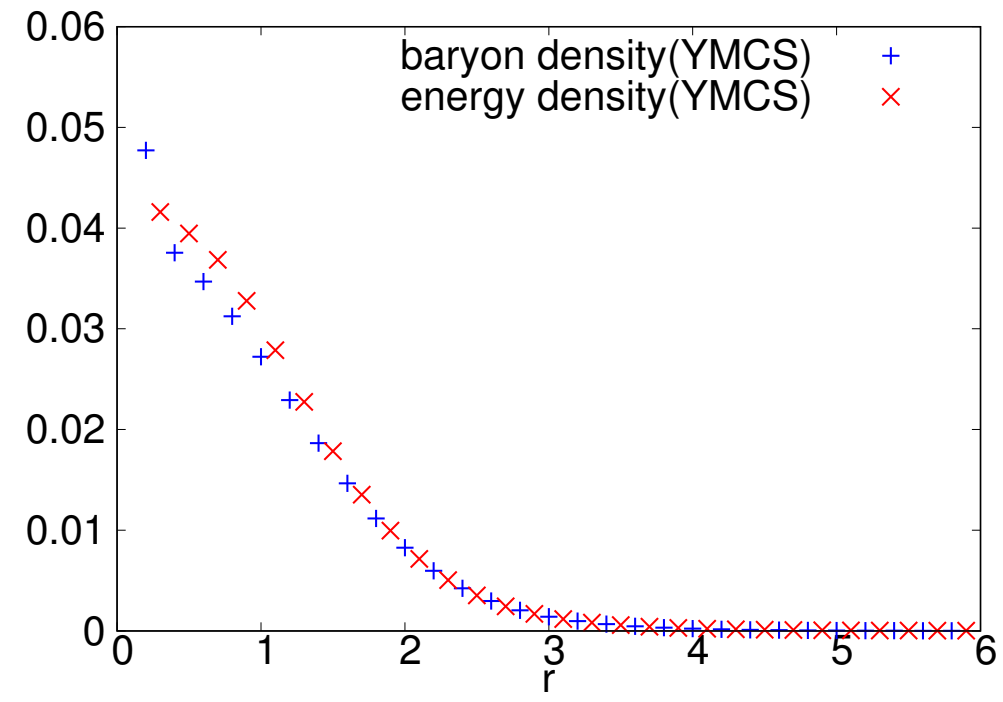

Figure 4. The energy density $\mathcal{E}(r)$ and the baryon-number density $\rho_{B}(r)$ inside the baryon as the function of $r$ in holographic QCD. In this figure, the unit of $M_{\mathrm{KK}}=1$ is taken, and hence the physical unit of the horizontal axis is $M_{\mathrm{KK}}^{-1} \simeq 0.2 \mathrm{fm}$.

\section{Meron in holographic QCD and two-merons oscillation modes}

As a next new subject, we study a meron [19], which has a half topological charge, in holographic QCD for the first time.

The meron is a curious classical solution of the Euclidean four-dimensional YangMills theory, since it is zero-size "half-instanton" with the half topological charge $(Q=1 / 2)$ and has an infinite Euclidean action $(S=\infty)$. However, a recent study reports that such a meronic configuration can have a finite energy in the $1+4$ dimensional Yang-Mills theory in the presence of some background gravity like a black hole [20].

Then, we study meron configurations in $(x, y, z, w)$-space in $1+4$ dimensional $\mathrm{U}\left(N_{f}\right)$ holographic QCD, where the background gravity exists. It is notable that, as well as instantons, the meron can be considered in spatial $(x, y, z, w)$-coordinate space in $1+4$ dimensional $\mathrm{U}\left(N_{f}\right)$ holographic QCD with $N_{f} \geq 2$ even in the Minkowski metric, and such a meron configuration in holographic QCD corresponds to a half baryon-number object with $B=1 / 2$ in QCD.

Before proceeding merons in holographic QCD, we briefly summarize traditional merons in the four-dimensional Euclidean Yang-Mills theory, where the space-time is Euclidean and it is difficult to consider Minkowski-time evolution of merons.

In the four-dimensional Euclidean $\mathrm{SU}(2)$ Yang-Mills theory, the single meron is expressed by

$$
A_{\mu}^{a}=-\frac{1}{2} \eta_{\mu \nu}^{a} \frac{x_{\nu}}{x^{2}}
$$

where $\eta_{\mu \nu}^{a}$ denotes the 't Hooft symbol [19]. For the meron, the topological charge $Q$ is one half:

$$
Q \equiv \frac{1}{16 \pi^{2}} \int d^{4} x \operatorname{tr}\left(F_{\mu \nu} \tilde{F}_{\mu \nu}\right)=\frac{1}{2}
$$


On the other hand, the action of the single meron is infinite in the infinite volume [19], and gradually increases as $\ln L$ for the system with the size $L^{4}$ :

$$
S_{L} \equiv \frac{1}{16 \pi^{2}} \int^{L} d^{4} x \operatorname{tr}\left(F_{\mu \nu} F_{\mu \nu}\right) \sim 3 \pi^{2} \ln L .
$$

It is notable that divergence of the meron action is logarithmic and weak [19]. Then, its divergence or finiteness is nontrivial in the presence of background gravity.

Now, we proceed merons in 1+4 dimensional holographic QCD. Note here that holographic QCD already has four spatial coordinates $(x, y, z, w)$ and merons can be introduced in this four-dimensional space, without necessity of the Euclidean process. Furthermore, we can consider the Minkowski-time $(t)$ evolution of merons in $1+4$ dimensional holographic QCD, unlike the four-dimensional Euclidean Yang-Mills theory.

\subsection{Single meron in holographic $Q C D$}

To begin with, we consider a single meron in $1+4$ dimensional $\mathrm{U}\left(N_{f}\right)$ holographic QCD with $N_{f}=2$. First, we consider a smeared-type meron configuration [19] in spatial coordinates $(x, y, z, w)$ in holographic QCD:

$$
A_{M}^{a}=-\frac{1}{2} \eta_{M N}^{a} \frac{x_{N}}{x^{2}+a^{2}}
$$

with the meron size $a$. Here, the capital indices $M, N$ denote the spatial coordinates $(x, y, z, w)$, and the extra coordinate $w$ plays a role of Euclidean time.

For the meron configuration, we consider the energy at the leading of $1 / N_{c}$ and $1 / \lambda$ :

$$
E_{5 \mathrm{YM}}^{\mathrm{SU}\left(N_{f}\right)}=\kappa \int d^{3} x d w \operatorname{tr}\left[\frac{1}{2} h(w) F_{i j}^{2}+k(w) F_{k w}^{2}\right],
$$

where the first and second terms can be regarded as the "magnetic energy" and the "electric energy", respectively, when $w$ is regarded as a Euclidean time.

As the result, we find that the "magnetic energy" of the meron configuration becomes finite, owing to the reduction gravitational factor $h(w)$, but the "electric energy" of the meron is more strongly divergent, due to the increasing gravitational factor $k(w)$. Even if one includes the CS term of the next leading of $1 / \lambda$, the total energy of the meron configuration is infinite, because the additional energy is repulsive and positive.

Next, we numerically study the single meron solution and its energy in holographic QCD, using the technique in the previous section. In fact, we consider the $\mathrm{SO}(3)$ rotational-invariant meron configuration, where holographic QCD is generally reduced into $1+2$ dimensional Abelian Higgs theory. We numerically examine the single-meron solution with the half topological charge $Q=B=1 / 2$ in the Abelian Higgs theory i) at the leading order and ii) up to the next leading order of $1 / \lambda$ including the CS term, respectively. For both cases, we find that the energy of the single meron solution with $Q=1 / 2$ is divergent when the volume goes to infinite. 
In fact, there is no single meron with a finite energy in holographic QCD, which means natural absence of such a half baryon-number object with $B=1 / 2$ in QCD.

\subsection{Meron-pair oscillation in holographic QCD}

Finally, we study the two-merons configuration and its time-dependent oscillation in the extra fifth-direction in holographic QCD, as is schematically depicted in Fig.5. The two-merons configuration is topologically identified as a kind of a single instanton, and the time-dependent oscillation can be regarded as an excitation of a single baryon in holographic QCD.

We consider the two smeared merons with the same size $a$ and their centers being located at $x_{1}=(0,0,0, l(t))$ and $x_{2}=(0,0,0,-l(t))$ in the $(x, y, z, w)$-space,

$$
\begin{aligned}
A_{M}^{a} & =-\frac{1}{2} \eta_{M N}^{a}\left[\frac{\left(x-x_{1}\right)_{N}}{\left(x-x_{1}\right)^{2}+a^{2}}+\frac{\left(x-x_{2}\right)_{N}}{\left(x-x_{2}\right)^{2}+a^{2}}\right] \\
& =-\frac{1}{2} \eta_{M N}^{a}\left[\frac{\{x-l(t) \hat{w}\}_{N}}{\{x-l(t) \hat{w}\}^{2}+a^{2}}+\frac{\{x+l(t) \hat{w}\}_{N}}{\{x+l(t) \hat{w}\}^{2}+a^{2}}\right],
\end{aligned}
$$

where $\hat{w}$ denotes the unit vector in the $w$-direction and $M, N$ take $(x, y, z, w)$.

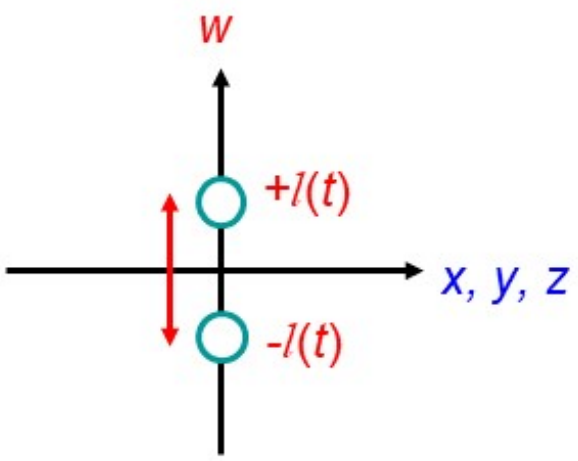

Figure 5. A schematic figure of a new-type baryon excitation of two smeared-merons oscillation in the extra fifth-direction in holographic QCD. The oscillation energy of the meron-pair is finite. Since this oscillation is in the extra $w$-direction, which is independent of ordinary coordinates, $x, y, z$, this type of oscillating excitation is expected to appear for any baryon universally, as an interesting possibility.

Note that, when the two centers of merons coincide as $l=0$, this two-merons system becomes an instanton with the size $a$, which corresponds to a single baryon in holographic QCD. In fact, this two-merons configuration is a special deformation of an instanton, and the two-merons oscillation in holographic QCD can be regarded as a special excitation of a single baryon. Then, the two-merons system has a finite energy like an instanton in holographic QCD. Here, we take the meron size to be $a \simeq 0.4 \mathrm{fm}$, corresponding to the instanton size describing the ground-state baryon in holographic QCD up to the next leading of $1 / \lambda$, as was shown in the previous section.

To get an analytical rough estimate for the two-merons oscillation energy, we consider $S_{5 \mathrm{YM}}$, the leading term on $1 / N_{c}$ and $1 / \lambda$, and use an approximation of 
$h(w)=k(w)=1$. After a lengthy calculation, we obtain the Lagrangian for $l(t)$ up to $O\left(l^{4}\right)$ :

$$
L[l(t)]=\frac{1}{2} m l^{2} l^{2}-\frac{1}{2} k l^{2}-\frac{1}{8} m \omega^{2} l^{4},
$$

with the coefficients of

$$
m=\frac{216}{5} \pi^{2} \kappa a^{-2}, \quad k=\frac{12}{5} \pi^{2} \kappa a^{-2}, \quad \omega=\frac{1}{6} \sqrt{\frac{202}{7}} a^{-1} \simeq 0.9 a^{-1} .
$$

By changing the variable $q \equiv \frac{1}{2} l^{2}$, we find

$$
L=\frac{1}{2} m \dot{q}^{2}-k q-\frac{1}{2} m \omega^{2} q^{2} .
$$

Thus, the oscillation energy of this meron-pair is finite, and its rough estimate at the semi-classical level is about $\omega \simeq 450 \mathrm{MeV}$ for $a \simeq 0.4 \mathrm{fm}$ for the lowest excitation mode, although there is some uncertainty on the boundary condition at $q=0$.

Because of the oscillation in the extra $w$-direction, all the quantum numbers of this-type excited baryons are just the same as the ground-state baryon. Then, as a possibility, this excitation might correspond to the Roper resonance N*(1440).

Note that this oscillation is in the extra $w$-direction, which is independent of

ordinary coordinates $(x, y, z)$. Then, as an interesting conjecture, this type of oscillating excitation in the extra $w$-direction might universally appear for any baryon, keeping the same quantum numbers.

Such an oscillation mode in the extra $w$-direction is easily grasped in the holographic description, although it would be described as a highly complicated collective mode in QCD without viewpoint of the extra direction.

\section{Summary and Concluding Remarks}

In this paper, we have studied topological objects in holographic QCD in the two-flavor $\left(N_{f}=2\right)$ case based on the Sakai-Sugimoto model, which is infrared equivalent to $1+3$ dimensional massless QCD. Using the gauge/gravity duality, holographic QCD is described as $1+4$ dimensional $\mathrm{U}\left(N_{f}\right)$ gauge theory in flavor space with a background gravity, and its instanton solutions correspond to baryons.

First, we have reduced $1+4$ dimensional holographic QCD into a $1+2$ dimensional Abelian Higgs theory in a curved space using the Witten Ansatz, and have considered its topological aspect. We have numerically calculated the Abrikosov vortex solution and have investigated single baryon properties.

Second, we have studied a single meron and two merons in holographic QCD. We have found that the single meron carrying a half baryon number has an infinite energy also in holographic QCD. We have proposed a new-type baryon excitation of the twomerons oscillation in the extra-direction of holographic QCD.

In the end, we summarize in Fig.6 various topological description of baryons presented from holographic QCD: 
- In 1+4 dimensional $\mathrm{SU}\left(N_{f}\right)$ holographic QCD, baryons are described with instantons in four-dimensional space $(x, y, z, w)$, based on the nontrivial homotopy group $\Pi_{3}\left(\mathrm{SU}\left(N_{f}\right)\right)=\mathbf{Z}$.

- In the 1+2 dimensional Abelian Higgs theory reduced from holographic QCD, the baryon is expressed as the Abrikosov vortex in $(r, w)$-plane, based on $\Pi_{1}(\mathrm{U}(1))=\mathbf{Z}$.

- The baryon is also described as a topological chiral soliton in a $1+3$ dimensional generalized Skyrme model derived from holographic QCD, based on $\Pi_{3}\left(\mathrm{SU}\left(N_{f}\right)_{L} \times\right.$ $\left.\mathrm{SU}\left(N_{f}\right)_{R} / \mathrm{SU}\left(N_{f}\right)_{V}\right)=\mathbf{Z}$.

These different topological objects are mathematically related, and these topological charges are exactly identical. In this way, holographic QCD gives a new physical picture and deep insight for baryons in QCD.

\section{Various Topological Description of Baryons in QCD}

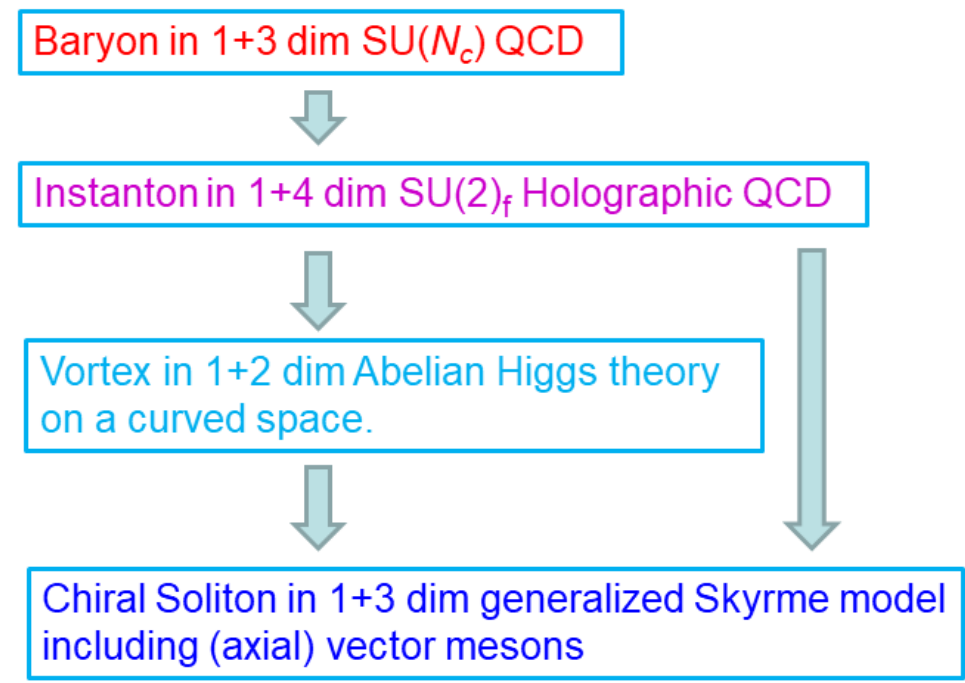

Figure 6. Various topological description of baryons in QCD. In 1+4 holographic QCD (HQCD), baryons are described with instantons in four-dimensional space $(x, y, z, w)$. The baryon is also expressed as the Abrikosov vortex in the $1+2$ Abelian Higgs theory reduced from HQCD. The baryon is eventually described as a topological chiral soliton in a $1+3$ generalized Skyrme model derived from HQCD. These different topological objects are mathematically related, and these topological charges are exactly identical.

\section{Acknowledgements}

We thank Prof. S. Sugimoto and Dr. T. Ishii for their useful comments and discussions. H.S. is supported by the Grants-in-Aid for Scientific Research [19K03869] from Japan Society for the Promotion of Science. 


\section{References}

[1] Polchinski J 1995 Dirichlet Branes and Ramond-Ramond charges Phys. Rev. Lett. 75, 4724-4727

Polchinski J 1998 String Theory (Cambridge: Cambridge University Press)

[2] Maldacena J M 1998 The Large N limit of superconformal field theories and supergravity Adv. Theor. Math. Phys. 2, 231-252.

[3] Witten E 1998 Anti-de Sitter space and holography Adv. Theor. Math. Phys. 2, 253-291

Witten E 1998 Anti-de Sitter space, thermal phase transition, and confinement in gauge theories Adv. Theor. Math. Phys 2, 505-532

[4] Sakai T and Sugimoto S 2005 Low energy hadron physics in holographic QCD Prog. Theor. Phys. 113, 843-882

Sakai T and Sugimoto S 2005 More on a holographic dual of QCD Prog. Theor. Phys. 114, 1083-1118

[5] Nawa K, Suganuma H and Kojo T 2007 Baryons in holographic QCD Phys. Rev. D75, 086003

[6] Hata H, Sakai T, Sugimoto S and Yamato S 2007 Baryons from instantons in holographic QCD Prog. Theor. Phys. 117, 1157-1180

[7] Hong D K, Rho M, Yee H U and Yi P 2007 Chiral Dynamics of Baryons from String Theory Phys. Rev. D76, 061901

Hong D K, Rho M, Yee H U and Yi P 2007 Dynamics of baryons from string theory and vector dominance JHEP 09, 063

[8] Hashimoto K, Sakai T and Sugimoto S 2008 Holographic Baryons: Static Properties and Form Factors from Gauge/String Duality Prog. Theor. Phys. 120, 1093-1137

[9] Nawa K, Suganuma H and Kojo T 2009 Brane-induced Skyrmion on $S^{3}$ : Baryonic matter in holographic QCD Phys. Rev. D79, 026005

[10] Cherman A and Ishii T 2012 Long-distance properties of baryons in the Sakai-Sugimoto model Phys. Rev. D86, 045011

[11] Bolognesi S and Sutcliffe P 2014 The Sakai-Sugimoto soliton JHEP 01, 078

[12] Rozali M, Stang J B and van Raamsdonk M 2014 Holographic Baryons from Oblate Instantons JHEP 02, 044

[13] Matsumoto K, Nakagawa Y and Suganuma H 2017 A Study of the H-dibaryon in Holographic QCD JPS Conf. Proc. 13, 020014

Suganuma H and Matsumoto K 2017 Holographic QCD for H-dibaryon (uuddss) EPJ Web Conf. 137, 13018

[14] Skyrme T H R 1961 A Nonlinear field theory Proc. Roy. Soc. A260, 127-138

Skyrme T H R 1962 A Unified Field Theory of Mesons and Baryons Nucl. Phys. 31 556-569

[15] Witten E 1979 Baryons in the 1/N Expansion Nucl. Phys. B160, 57-115

[16] Adkins G, Nappi C R and Witten E 1983 Static Properties of Nucleons in the Skyrme Model Nucl. Phys. B228 552-566

[17] Witten E 1998 Baryons and branes in anti-de Sitter space JHEP 07, 006

[18] Witten E 1977 Some Exact Multi - Instanton Solutions of Classical Yang-Mills Theory Phys. Rev. Lett. 38, 121-124

[19] Callan C G, Dashen R and Gross D J 1979 A Mechanism for Quark Confinement Phys. Lett. B66, $375-381$

Callan C G, Dashen R and Gross D J 1979 A Theory of Hadronic Structure Phys. Rev. D19, 1826-1855

[20] Canfora F, Gomberoff A, Oh S H, Rojas F and Salgado-Rebolledo P 2019 Meronic Einstein-YangMills black hole in 5D and gravitational spin from isospin effect JHEP 06, 081 